\title{
A CHARACTERIZATION OF THE RADICAL OF AN ALGEBRA
}

\section{SAM PERLIS}

1. The first main result. We shall prove the following result.

THEOREM 1. Let $F$ be any field and $A$ an algebra over $F$ with a unity element. Then the radical of $A$ consists of all elements $h$ such that $g+h$ is regular for every regular $g$.

Let $H$ be the set of all elements $h$ defined in the theorem. It is easy to see that $H$ is a linear set over $F$. We shall prove now that if $A$ is simple, $H=0$.

Let $g$ and $g_{1}$ be any regular elements of $A$ and $h$ be in $H$. Then $g_{1}^{-1} g+h$ is regular so that $g+g_{1} h$ is regular. Hence $g_{1} h$ is in $H$ and similarly $h g_{1}$ is in $H$. An arbitrary element $a$ of $A$ has $^{1}$ the form $a=\sum_{i=1}^{n} g_{i}$ with regular elements $g_{i}$ so that $a h=\sum g_{i} h$ is a sum of elements $g_{i} h$ of $H$. Thus $a h$, and similarly $h a$, is in $H$ so that $H$ is an ideal of $A$. If $H \neq 0$ then $H=A$ since $A$ is simple. But $A$ contains the regular element -1 , and $(-1)+1$ is not regular so that 1 cannot be in $H$, whence $H \neq A$. Hence $H=0$.

Next we shall prove that $H=0$ whenever $A$ is semi-simple. Now $A=A_{1}+A_{2}+\cdots+A_{t}$ where the $A_{i}$ are simple, and each $x$ of $A$ has a unique expression $x=a_{1}+a_{2}+\cdots+a_{t}$ with $a_{i}$ in $A_{i}$. Further, $x$ is regular if and only if each $a_{i}$ is a regular element of $A_{i}$. Let $g=g_{1}+\cdots+g_{t}$ be regular, $h=h_{1}+\cdots+h_{t}$ be in $H$, so that $g+h=\left(g_{1}+h_{1}\right)+\cdots+\left(g_{t}+h_{t}\right)$. Then $g+h$ is regular for every regular $g$ if and only if $g_{i}+h_{i}$ is regular in $A_{i}$ for every regular $g_{i}$ of $A_{i}$. By the proof above for simple algebras every $h_{i}=0$ so that $h=0$ and $H=0$.

In considering the case of a general algebra $A$, we show first that the radical $R$ is contained in $H$. Let $g$ be regular and $r$ lie in $R$. Then $g+r$ is regular if and only if $1+g^{-1} r$ is regular. Now $g^{-1} r$ is in $R$, $\left(g^{-1} r\right)^{t}=0$ for some integer $t,\left(g^{-1} r\right)^{2 t+1}+1=1$. If $\lambda$ is an indeterminate, $\lambda+1$ is a factor of $\lambda^{2 t+1}+1$ so that $g^{-1} r+1$ is a factor of $\left(g^{-1} r\right)^{2 t+1}+1=1$; hence, $g^{-1} r+1$ is regular, $g+r$ is regular, $r$ is in $H$, and $R$ is contained in $H$.

It remains to prove that $R$ contains $H$. Since $A-R$ is semi-simple, the set $H_{0}$ defined for $A-R$, similarly to $H$ for $A$, is the zero set. If $g$ is regular in $A$ and $h$ is in $H$, the class $[g+h]$ in $A-R$ is a regular ele-

Presented to the Society, April 12, 1941; received by the editors April 11, 1941.

${ }^{1}$ K. Shoda, Mathematische Annalen, vol. 107 (1933), pp. 252-258. 
ment of $A-R$. But $[g+h]=[g]+[h]$, and $[g]$ varies over all ${ }^{2}$ regular elements of $A-R$ so that $[h]$ must be in $H_{0}=[0]=R$. Hence $h$ is in $R$, $H$ is contained in $R, H=R$, and the proof of the theorem is complete.

2. Extension to arbitrary algebras. The theorem above is applicable to algebras $A_{0}$ without a unity element in the sense that by adjoining a unity element to $A_{0}$ we do not alter its radical. To see this, let $R_{0}$ be the radical of $A_{0}$ and $R$ the radical of the corresponding algebra $A$ with a unity element. Every element of $A$ has the unique form $a=\alpha+a_{0}$ with $\alpha$ in $F, a_{0}$ in $A_{0}$. If $r_{0}$ is in $R_{0}$ then $a r_{0}=\alpha r_{0}+a_{0} r_{0}$ is a sum of elements of $R_{0}, a r_{0}$ is nilpotent, $r_{0}$ is properly nilpotent in $A$, so that $R_{0} \leqq R$. Conversely, let $r$ be in $R$ so that $r^{t}=0$ for some integer $t, r=\sigma+s_{0}$ with $\sigma$ in $F, s_{0}$ in $A_{0}, r^{t}=\sigma^{t}+s_{1}=0$ with $s_{1}$ in $A_{0}$. Then $s_{1}=0, \sigma=0$ so that $r=s_{0}$ in $A_{0}$. But $r$ is properly nilpotent in $A$, hence in $A_{0}$, hence $r$ is in $R_{0}, R \leqq R_{0}, R=R_{0}$.

While the fact just proved enables one to apply Theorem 1 to arbitrary algebras, nevertheless it is desirable to obtain a criterion not dependent on the unity element, as Professor Marshall Hall has pointed out to the author. The remainder of this section is devoted to this purpose.

If $A$ is an algebra without a unity element, the symbol $A^{\prime}$ will be used throughout the paper to denote the algebra obtained from $A$ by adjoining a unity element. If $A$ has a unity element, $A^{\prime}$ is defined to be $A$.

Definition. An element $x$ of an algebra will be called "quasi-regular" in case there is an element $y$ in the algebra such that

$$
x+x y+y=0,
$$

and then $y$ will be called the "quasi-inverse" of $x$.

Since $(1+x)(1+y)=1+x+x y+y$, we see at once that if an element $x$ of $A$ is quasi-regular in $A$, then $1+x$ is regular in $A^{\prime}$; and conversely, if $1+x$ is regular in $A^{\prime}$ for $x$ in $A$, then $x$ is quasi-regular in $A^{\prime}$, and actually in $A$ as the following result shows.

Lemma 1. Let $A$ be an algebra over $F$. If $A=A^{\prime}$, a quantity of $A$ is regular if and only if it has the form $1+x$ where $x$ is quasi-regular. If $A \neq A^{\prime}$, a quantity of $A^{\prime}$ is regular if and only if it is expressible as $\alpha(1+x)$ where $\alpha$ is a nonzero element of $F$ and $x$ is a quasi-regular element of $A$.

${ }^{2}$ For, if $[b]$ is any regular element of $A-R$, then $[b][c]=[1], b c=1+r$ with $r$ in $R$. Since we have already proved $R \leqq H$ it follows that $1+r$ is regular, $b c$ is regular, so that $b$ is regular. 
The case $A=A^{\prime}$ is completed by the remark above the statement of the lemma. In the case $A \neq A^{\prime}$ the same remark shows that if $x$ is quasi-regular in $A$, then $1+x$ is regular and so is $\alpha(1+x)$. Conversely, let $g=\alpha+x_{0}$ be any regular element of $A^{\prime}$ with $\alpha$ in $F$ and $x_{0}$ in $A$. We readily find that $\alpha \neq 0$ so that $g=\alpha(1+x), 1+x$ is regular, and

$$
(1+x)(\beta+y)=\beta+(\beta x+x y+y)=1
$$

for some $\beta$ in $F$ and $y$ in $A$. Since $\beta x+x y+y$ is in $A$ and $\beta$ is in $F$, we must have $\beta=1, x+x y+y=0$, so that $x$ is quasi-regular.

Observe that this lemma provides unique expressions for the regular quantities of $A^{\prime}$.

If $x$ is quasi-regular in $A$, its quasi-inverse is the unique element $y$ such that $1+y$ is the inverse of $1+x$. Moreover,

$$
(1+x)(1+y)=1=(1+y)(1+x),
$$

whence $x y=y x$. Finally, the inverse $1+y$ of the regular element $1+x$ is known to be a polynomial in $1+x$ so that $y$ is a polynomial in $x$.

Lemma 2. If $x$ is a quasi-regular element of an algebra $A$, its quasiinverse is unique, is a polynomial in $x$, and commutes with $x$.

We now obtain the following main criterion.

THEOREM 2. Let $A$ be an algebra over a field $F$. Then an element $r$ of $A$ is in the radical of $A$ if and only if $x+\alpha r$ is quasi-regular in $A$ for every $x$ of $A$ which is quasi-regular and every $\alpha$ of $F$.

If $x$ is quasi-regular and $r$ is in the radical, $\alpha r$ is in the radical of both $A$ and $A^{\prime}, 1+x$ is regular in $A^{\prime}$, and thus $1+x+\alpha r$ is regular by Theorem 1 . By Lemma 1 the element $x+\alpha r$ of $A$ must be quasiregular. Conversely, suppose that $r$ is an element of $A$ with the property stated in the theorem. Any regular element $g$ of $A^{\prime}$ has the form $g=\alpha+\alpha x, \alpha \neq 0$ in $F$, and $x$ quasi-regular in $A$. By hypothesis $x+\alpha^{-1} r$ is quasi-regular so that

$$
h=1+x+\alpha^{-1} r
$$

is regular, and $\alpha h=g+r$ is regular. By Theorem 1 the element $r$ is in the radical of $A^{\prime}$, hence in the radical of $A$.

A common characterization of the radical is that it consists of zero and all properly nilpotent elements. It may be noticed that this characterization is strongly in contrast with the present ones which are phrased in terms of addition rather than multiplication and are concerned with preserving regularity rather than nilpotency. 
3. Some applications. As an application let us consider bound algebras which have been studied by M. Hall. ${ }^{3}$ In his Theorem 3.6, Hall found that a bound algebra $A$ contains three pairwise orthogonal idempotents $e_{1}, e_{2}, e_{3}$ with the following properties. If $A_{i}=e_{i} A e_{i}$ $(i=1,2,3)$ and $R$ is the radical of $A$, then

(1) $A$ is the supplementary sum $A=A_{1}+A_{2}+\left(A_{3}, R\right)$;

(2) $R e_{1}=0, e_{2} R=0$;

(3) $A_{1}$ and $A_{2}$ are semi-simple.

We shall prove:

I. If $A$ has a unity element $e$, then $e=e_{1}+e_{2}+e_{3}$.

For proof, let $e_{0}=e-\left(e_{1}+e_{2}+e_{3}\right)$ so that $e_{0}$ is either zero or an idempotent orthogonal to each $e_{i}$. By property (1) we have $e_{0}=a_{1}+a_{2}$ $+\left(a_{3}+r\right)$ with $a_{i}$ in $A_{i}, r$ in $R$, and we may always assume, without loss of generality, that either $a_{3}=0$ or else $a_{3}$ is not in $R$. By the orthogonality of the $e_{i}$ and property (2) we have $e_{0} e_{3}=0=a_{1}, e_{2} e_{0}=0=a_{2}$. Now $e_{0}=a_{3}+r, e_{0} e_{3}=0=a_{3}+r e_{3}, a_{3}=-r e_{3}$ in $R$ so that $a_{3}=0$. Then $e_{0}=r$ in $R$ so that $e_{0}$ cannot be idempotent and we have $e_{0}=0$.

Next we again assume that $A$ has a unity element and prove:

II. If $x=a_{1}=a_{2}+\left(a_{3}+r\right)$ is any element of $A, a_{i}$ in $A_{i}, r$ in $R$, then $x$ is regular if and only if each $a_{i}$ is regular in $A_{i}$.

Since $r$ is in $R, x$ is regular if and only if $y=a_{1}+a_{2}+a_{3}$ is regular. If $z=b_{1}+b_{2}+\left(b_{3}+s\right)$ is an element of $A, b_{i}$ in $A_{i}, s$ in $R$, then

$$
y z=a_{1} b_{1}+a_{2} b_{2}+a_{3} b_{3}+s_{1}, \quad s_{1} \text { in } R,
$$

and $y z=e=e_{1}+e_{2}+e_{3}$ if and only if $a_{1} b_{1}=e_{1}, a_{2} b_{2}=e_{2}, a_{3} b_{3}=e_{3}-s_{1}$. Hence $a_{1}$ and $a_{2}$ must be regular in their respective algebras. The third equation above shows that $s_{1}$ must be in $A_{3}$ as well as in $R$, hence in the radical of $A_{3}$. Then $e_{3}-s_{1}$ is a regular element of $A_{3}, a_{3} b_{3}$ is regular in $A_{3}$, so that $a_{3}$ likewise must be regular in $A_{3}$. Conversely, if each $a_{i}$ has an inverse $b_{i}$ in $A_{i}$, it is clear that $b_{1}+b_{2}+b_{3}$ is the inverse of $a_{1}+a_{2}+a_{3}$.

An analogue of II not requiring a unity element will now be obtained.

III. Let $x=a_{1}+a_{2}+\left(a_{3}+r\right)$ be an element of a bound algebra $A$. Then $x$ is quasi-regular if and only if each $a_{i}$ is quasi-regular in $A_{i}$.

${ }^{3}$ The position of the radical in an algebra, Transactions of this Society, vol. 48 (1940), pp. 391-404. A "bound algebra" $A$ with radical $R$ is an algebra with the property that if $x R=R x=0$ for $x$ in $A$, then $x$ is in $R$. Hall has reduced the structure theory of arbitrary algebras to that of bound algebras and semi-simple algebras. 
By Theorem 2 the element $x$ is quasi-regular if and only if $x-r=y$ $=a_{1}+a_{2}+a_{3}$ is quasi-regular. If each $a_{i}$ has a quasi-inverse $b_{i}$ in $A_{i}$, then $z_{0}=b_{1}+b_{2}+b_{3}$ has the property that $y z_{0}=a_{1} b_{1}+a_{2} b_{2}+a_{3} b_{3}$ so that $y+y z_{0}+z_{0}=0$, and $y$ is quasi-regular. Conversely, suppose that $y$ has a quasi-inverse $z=b_{1}+b_{2}+\left(b_{3}+s\right)$ with $s$ in $R$ and $b_{i}$ in $A_{i}$. Then

$$
\begin{gathered}
y+y z+z=0=\sum_{i=1}^{3}\left(a_{i}+a_{i} b_{i}+b_{i}\right)+y s+s, \\
a_{3}+a_{3} b_{3}+b_{3}+y s+s=0, \quad a_{i}+a_{i} b_{i}+b_{i}=0
\end{gathered}
$$

for $i=1,2$. Thus $a_{1}$ and $a_{2}$ are quasi-regular in $A_{1}$ and $A_{2}$, respectively, and the quantity

$$
c=a_{3}+a_{3} b_{3}+b_{3}=-s-y s
$$

is in $A_{3}, e_{3} c=c=-e_{3} s-e_{3} y s=-e_{3} s-a_{3} s=-\left(e_{3}+a_{3}\right) s$. Since $y$ commutes with its quasi-inverse (Lemma 2), we have $y+z y+z=0$ and as in (4) we are led to the equation

$$
c^{\prime}=a_{3}+b_{3} a_{3}+b_{3}=-s-s y .
$$

Hence $c^{\prime}=c^{\prime} e_{3}=-s\left(e_{3}+a_{3}\right)$. We have proved

$$
c=-\left(e_{3}+a_{3}\right) s, \quad c^{\prime}=-s\left(e_{3}+a_{3}\right) .
$$

Now $\left(e_{3}+a_{3}\right)\left(e_{3}+b_{3}\right)=e_{3}+c=e_{3}-\left(e_{3}+a_{3}\right) s$ so that

$$
\left(e_{3}+a_{3}\right)\left(e_{3}+b_{3}+s\right)=e_{3} .
$$

Likewise, by forming $\left(e_{3}+b_{3}\right)\left(e_{3}+a_{3}\right)=e_{3}+c^{\prime}$ we find

$$
\left(e_{3}+b_{3}+s\right)\left(e_{3}+a_{3}\right)=e_{3} \text {. }
$$

Since $e_{3}$ is the unity element of $A_{3}$, the results (7) and (8) show that the element $e_{3}+a_{3}$ of $A_{3}$ is not a divisor of zero in $A_{3}$. Thus $e_{3}+a_{3}$ is a regular element of $A_{3}$ so that $a_{3}$ must be quasi-regular. This completes the proof.

One may observe that property (3) is an immediate consequence of the fact III and Theorem 2. Suppose that $r_{1}$ is in the radical of $A_{1}$ and $x=a_{1}+a_{2}+\left(a_{3}+r\right)$ is any quasi-regular element of $A$. Then $a_{1}$ is quasi-regular in $A_{1}, a_{1}+\alpha r_{1}$ is quasi-regular in $A_{1}$ for every $\alpha$ of $F$, and $x+\alpha r_{1}=\left(a_{1}+\alpha r_{1}\right)+a_{2}+\left(a_{3}+r\right)$ has the requisite form for a quasiregular element of $A$. By Theorem 2 the quantity $r_{1}$ is in $R$ as well as in $A_{1}$. But then $r_{1}=0$ by property (1). Thus $A_{1}$ is semi-simple, and similarly $A_{2}$ is semi-simple.

UNIVERSITY OF MiCHIGAN 